\title{
Convergence and supercloseness in a balanced norm of finite element methods on Bakhvalov-type meshes for reaction-diffusion problems $^{\star}$
}

\author{
Jin Zhanga,*, Xiaowei Liu ${ }^{\mathrm{b}, 1}$ \\ ${ }^{a}$ School of Mathematics and Statistics, Shandong Normal University, Jinan 250014, China \\ ${ }^{b}$ School of Mathematics and Statistics, Qilu University of Technology (Shandong Academy of Sciences), \\ Jinan 250353, China
}

\begin{abstract}
In convergence analysis of finite element methods for singularly perturbed reactiondiffusion problems, balanced norms have been successfully introduced to replace standard energy norms so that layers can be captured. In this article, we focus on the convergence analysis in a balanced norm on Bakhvalov-type rectangular meshes. In order to achieve our goal, a novel interpolation operator, which consists of a local weighted $L^{2}$ projection operator and the Lagrange interpolation operator, is introduced for a convergence analysis of optimal order in the balanced norm. The analysis also depends on the stabilities of the $L^{2}$ projection and the characteristics of Bakhvalov-type meshes. Furthermore, we obtain a supercloseness result in the balanced norm, which appears in the literature for the first time. This result depends on another novel interpolant, which consists of the local weighted $L^{2}$ projection operator, a vertices-edges-element operator and some corrections on the boundary.
\end{abstract}

Keywords: Singular perturbation, Reaction-diffusion equation, Bakhvalov-type mesh, Finite element method, Balanced norm, Supercloseness.

*This research is partially supported by National Natural Science Foundation of China $(11771257,11601251)$

*Corresponding author: jinzhangalex@hotmail.com

${ }^{1}$ Email: xwliuvivi@hotmail.com 


\section{Introduction}

In this article we consider the singularly perturbed reaction-diffusion equation

$$
\begin{aligned}
-\varepsilon^{2} \Delta u+b u=f & \text { in } \Omega:=(0,1)^{2}, \\
u=0 & \text { on } \partial \Omega,
\end{aligned}
$$

where $\varepsilon$ is a positive parameter. Assume that $b$ and $f$ are sufficiently smooth and

$$
b(x, y) \geqslant 2 \beta^{2}>0 \quad \forall(x, y) \in \bar{\Omega},
$$

with a positive constant $\beta$. Under these conditions on the data in problem (1.1), there exists a unique solution in $H_{0}^{1}(\Omega) \cap H^{2}(\Omega)$ for all $f \in L^{2}(\Omega)$. The solution to problem (1.1) typically exhibits boundary layers of width $\mathcal{O}(\varepsilon|\ln \varepsilon|)$ along all of $\partial \Omega$ in the singularly perturbed case $0<\varepsilon \ll 1$ of interest.

For singularly perturbed problems, it is popular to introduce layer-adapted meshes [14, 19, 20] to fully resolve layers. Then uniform convergence with respect to singular perturbation parameter can be achieved for standard numerical methods. There are two kinds of layer-adapted meshes widely used in the literature, which are Bakhvalov-type mesh (B-mesh) and Shishkin-type mesh (S-mesh) (see [14]). There are a lot of research results on convergence theories of finite element methods on S-meshes; see [19, 7, 6, 22, 23, 27, 16] and references therein.

Although B-meshes usually have better performances than S-meshes, there are very few articles on uniform convergence of finite element methods on the former. One main reason is that B-meshes have specific transition points between the fine and coarse parts, which are independent of mesh parameter. In the meantime, they bring great difficulties to convergence analysis. For example, Lagrange interpolant does not work well for Bmeshes. Recently, Zhang and Liu [25, 26] proposed an variant of Lagrange interpolant for finite element methods on B-meshes in the case of convection-diffusion equations and succeeded to obtain a uniform convergence of optimal order. 
For reaction-diffusion problems, they also proved optimal order of uniform convergence in the natural energy norm in [24]. However, energy norm is not strong enough to capture layers as the singular perturbation parameter tends to zero. Thus balanced norms, which are stronger than standard energy norms and characterize layers in an more appropriate way, were introduced in [13] for a mixed finite element method and [18] for a finite element method. The authors [18] introduced an $L^{2}$-projection to obtain desired estimations by $L^{\infty}$-stability of the $L^{2}$-projection on Shishkin mesh. To improve estimations in [18], the authors [8] introduced a new interpolation, which consists a local weighted $L^{2}$ projection defined on the uniform part of S-meshes. Unfortunately, unlike S-meshes, there is little development on convergence theories in balanced norms on B-meshes.

In this manuscript we analyze convergence theories in the balanced norm introduced in [18] for $k$ th $(k \geqslant 1)$ order finite element method on Bakhvalov-type rectangular meshes. For this purpose, we propose a novel interpolant according to the structures of B-meshes and layer functions. This interpolant consists of a local weighted $L^{2}$ projection defined on a proper mesh subdomain and the Lagrange interpolant. To prove the convergence of optimal order in the balanced norm, we must take into account the scales of the meshes and different stabilities of the $L^{2}$ projection. The optimal order convergence is also supported by our numerical experiments. Furthermore, we propose another novel interpolant operator, which consists of the local weighted $L^{2}$ projection operator, a vertices-edgeselement operator and some corrections on the boundary. By careful derivations, we obtain a supercloseness result, which appears in the literature for the first time. Here "supercloseness" means that the convergence order for the error between some interpolation of the solution $u$ and the numerical solution $u^{N}$ in some norm is greater than the order for $u-u^{N}$ in the same norm.

The rest of the paper is organized as follows. In Section 2 we present a priori information of the solution to (1.1), then introduce Bakhvalov-type meshes, finite element methods and some preliminary results. In Section 3 we give a new interpolant and prove 
uniform convergence of optimal order in the balanced norm. Supercloseness result is given in Section 4 by means of another novel interpolant. In Section 5, numerical results illustrate our theoretical results.

Let $D \subset \Omega$. In this article, we will write $(\cdot, \cdot)_{D}$ for the inner product in $L^{2}(D),\|\cdot\|_{D}$, $\|\cdot\|_{\infty, D},\|\cdot\|_{1, D}$ and $|\cdot|_{1, D}$ for the standard norms in $L^{2}(D), L^{\infty}(D), L^{1}(D)$ and the standard seminorm in $H^{1}(D)$, respectively. If $D=\Omega$, the subscript will be omitted from the above norm designations. Throughout the paper, all constants $C$ and $C_{i}$ are independent of $\varepsilon$ and $N$; the constants $C$ are generic while subscripted constants $C_{i}$ are fixed.

\section{Finite element method on Bakhvalov-type mesh}

\subsection{Regularity results}

To construct layer-adapted meshes and analyze uniform convergence, we need a priori information of the solution $u$ to (1.1), such as pointwise estimations of the derivatives of the solution, the locations and widths of layers.

For this aim, we give the following assumption on the solution $u$ to (1.1) according to $[9,15,3]$.

Assumption 1. The solution $u$ of (1.1) can be decomposed as

$$
u=v_{0}+\sum_{i=1}^{4} w_{i}+\sum_{i=1}^{4} z_{i} \quad \forall \boldsymbol{x} \in \bar{\Omega},
$$

where $v_{0}$ is the regular part, each $w_{i}$ is a boundary layer function and each $z_{i}$ is a corner layer function. For $k \in \mathbb{N}$ and $k \geqslant 1$, there exists a constant $C$ such that

$$
\begin{aligned}
& \left|\partial_{x}^{m} \partial_{y}^{n} v_{0}(x, y)\right| \leqslant C\left(1+\varepsilon^{(k+1)-m-n}\right) \quad \text { for } 0 \leqslant m+n \leqslant k+3, \\
& \left|\partial_{x}^{m} \partial_{y}^{n} w_{1}(x, y)\right| \leqslant C\left(1+\varepsilon^{(k+1)-m}\right) \varepsilon^{-n} e^{-\beta y / \varepsilon} \quad \text { for } 0 \leqslant m+n \leqslant k+2, \\
& \left|\partial_{x}^{m} \partial_{y}^{n} z_{1}(x, y)\right| \leqslant C \varepsilon^{-(m+n)} e^{-\beta(x+y) / \varepsilon} \quad \text { for } 0 \leqslant m+n \leqslant k+2
\end{aligned}
$$

and similarly for the remaining terms. Here denote $\frac{\partial^{m+n} v}{\partial x^{m} \partial y^{n}} b y \partial_{x}^{m} \partial_{y}^{n} v$.

In the following analysis, we will denote $\sum_{i=1}^{4} w_{i}+\sum_{i=1}^{4} z_{i}$ by $w$. 


\subsection{Bakhvalov-type meshes}

Two Bakhvalov-type meshes will be discussed. Let $N \in \mathbb{N}$ be divisible by 4 . The first Bakhvalov-type mesh is introduced in [17] and defined by

$$
x_{i}=y_{i}=\psi(i / N)= \begin{cases}-\frac{\sigma \varepsilon}{\beta} \ln (1-4(1-\varepsilon) i / N) & \text { for } i=0, \ldots, N / 4, \\ d_{1}(i / N-1 / 4)+d_{2}(i / N-3 / 4) & \text { for } i=N / 4, \ldots, 3 N / 4, \\ 1+\frac{\sigma \varepsilon}{\beta} \ln (1-4(1-\varepsilon)(1-i / N)) & \text { for } i=3 N / 4, \ldots, N,\end{cases}
$$

where $\sigma$ will be defined later and $d_{1}, d_{2}$ are used to ensure the continuity of $\psi(t)$ at $t=1 / 4$ and $t=3 / 4$. The second Bakhvalov-type mesh is introduced in [10, 11] and its mesh generating function is

$$
\varphi(t)= \begin{cases}-\frac{\sigma \varepsilon}{\beta} \ln (1-4 t) & \text { for } t \in[0, \vartheta] \\ d_{3}(t-\vartheta)+d_{4}(t-1+\vartheta) & \text { for } t \in(\vartheta, 1-\vartheta), \\ 1+\frac{\sigma \varepsilon}{\beta} \ln (1-4(1-t)) & \text { for } t \in[1-\vartheta, 1]\end{cases}
$$

where $\sigma$ will be specified later, $\vartheta=1 / 4-C_{1} \varepsilon$ with some positive constant $C_{1}$ independent of $\varepsilon$ and $N, d_{3}$ and $d_{4}$ are chosen so that $\varphi(t)$ is continuous at $t=\vartheta$ and $t=1-\vartheta$. The original Bakhvalov mesh [1] can be recovered from (2.6) by setting $\vartheta=1 / 4-\mathcal{C}(\varepsilon) \varepsilon$ with $0<C_{2} \leqslant \mathcal{C}(\varepsilon) \leqslant C_{3}$.

Assumption 2. Assume that $\varepsilon \leqslant \min \left\{\frac{\beta}{4 \sigma}, 1\right\} N^{-1}$ in our analysis. In practice it is not a restriction.

For technical reasons, we also assume

$$
\frac{\sigma}{4 e \beta} \leqslant C_{1} \leqslant \frac{1}{2} \max \left\{\frac{\sigma}{\beta}, \frac{1}{4}\right\}
$$

Assume $N \geqslant \max \left\{8,2 \ln \frac{\sigma}{\beta}\right\}$. Under Assumption 2 and (2.7), we have $1 / 4-N^{-1} \leqslant \vartheta<1 / 4$ and $x_{N / 4} \leqslant 1 / 4$ for meshes (2.5) and (2.6). The location of $\vartheta$ and conditions imposed on 
$N$ and $C_{1}$ will simplify our later analysis without changing the essential difficulties in our analysis.

The mesh points are $x_{i}=y_{i}=\psi(i / N)$ or $x_{i}=y_{i}=\varphi(i / N)$ for $i=0,1, \ldots, N$. By drawing lines parallel to the axis through mesh points $\left\{\left(x_{i}, y_{j}\right)\right\}$, we obtain a Bakhvalovtype rectangular mesh with equidistant cells in the coarse region $\Omega_{0}=\left(x_{N / 4}, x_{3 N / 4}\right)^{2}$ and anisotropic cells in the layer region $\Omega \backslash \Omega_{0}$. The triangulation is denoted by $\mathcal{T}^{N}$. Denote by $\tau_{i, j}$ for the element $\left[x_{i}, x_{i+1}\right] \times\left[y_{j}, y_{j+1}\right]$ and by $\tau$ for a generic rectangular element, which dimensions are written as $h_{x, \tau}$ and $h_{y, \tau}$. Define $h_{i}:=x_{i+1}-x_{i}=y_{i+1}-y_{i}$ for $0 \leqslant i \leqslant N-1$.

In the following lemma, we collect some important properties possessed by Bakhvalovtype meshes, which are important for convergence analysis. The reader is referred to [25] for the detailed proof.

Lemma 1. Let Assumption圆 hold true. On Bakhvalov-type mesh (2.5) or (2.6), one has

$$
\begin{aligned}
& h_{0} \leqslant h_{1} \leqslant \ldots \leqslant h_{N / 4-2}, \\
& \frac{\sigma}{4 \beta} \varepsilon \leqslant h_{N / 4-2} \leqslant \frac{\sigma}{\beta} \varepsilon, \\
& C \varepsilon \leqslant h_{N / 4-1} \leqslant C N^{-1}, \\
& C_{4} N^{-1} \leqslant h_{i} \leqslant C_{5} N^{-1} \quad N / 4 \leqslant i \leqslant N / 2, \\
& x_{N / 4-1}=x_{3 N / 4+1} \geqslant C \sigma \varepsilon \ln N, \quad x_{N / 4}=x_{3 N / 4} \geqslant C \sigma \varepsilon \ln (1 / \varepsilon) .
\end{aligned}
$$

Note $h_{i}=h_{N-1-i}$ for $i=0,1, \ldots, N / 2$.

Let $i^{*}=j^{*}=N / 4-2$. Then one has

$$
h_{i}^{\mu} e^{-\beta x_{i} / \varepsilon} \leqslant C \varepsilon^{\mu} N^{-\mu} \quad \text { for } 0 \leqslant i \leqslant i^{*} \text { and } 0 \leqslant \mu \leqslant \sigma .
$$

Similar bounds hold for the variable $y$. 


\subsection{Finite element method}

Now we present the finite element method for problem (1.1). First, the weak form of problem (1.1) is written as

$$
\left\{\begin{array}{l}
\text { find } u \in V \text { such that for all } v \in V \\
a(u, v):=\varepsilon^{2}(\nabla u, \nabla v)+(b u, v)=(f, v),
\end{array}\right.
$$

with $V:=H_{0}^{1}(\Omega)$. The natural energy norm derived from $a(\cdot, \cdot)$ is

$$
\|v\|_{\varepsilon}:=\left(\varepsilon^{2}|v|_{1}^{2}+\|v\|^{2}\right)^{1 / 2}
$$

The bilinear form $a(\cdot, \cdot)$ is coercive with respect to this energy norm, i.e.,

$$
a(v, v) \geqslant \min \left\{2 \beta^{2}, 1\right\}\|v\|_{\varepsilon}^{2} \quad \forall v \in V
$$

From the Lax-Milgram lemma, the weak formulation (2.14) has a unique solution.

Let $\mathcal{Q}_{k}(\tau)$ denote $k$ th rectangular finite element. We introduce the finite element spaces

$$
V^{N}:=\left\{v \in H^{1}(\Omega):\left.v\right|_{\tau} \in \mathcal{Q}_{k}(\tau) \quad \forall \tau \in \mathcal{T}^{N}\right\} \text { and } V_{0}^{N}:=V^{N} \cap H_{0}^{1}(\Omega)
$$

Clearly, $V^{N}, V_{0}^{N} \subset V$. When we replace the infinite dimensional space $V$ with the finite dimensional space $V_{0}^{N}$, we get the $k$ th order finite element method

$$
\left\{\begin{array}{l}
\text { find } u^{N} \in V_{0}^{N} \text { such that for all } v_{0}^{N} \in V^{N} \\
a\left(u^{N}, v^{N}\right)=\left(f, v^{N}\right) .
\end{array}\right.
$$

Also, it is easy to very the coercivity

$$
a\left(v^{N}, v^{N}\right) \geqslant C\left\|v^{N}\right\|_{\varepsilon}^{2} \quad \forall v^{N} \in V_{0}^{N}
$$

and the Galerkin orthogonality

$$
a\left(u-u^{N}, v^{N}\right)=0 \quad \forall v^{N} \in V_{0}^{N},
$$


where (2.14) and (2.16) have been used. Furthermore, we introduce the balanced norm in [18], which is defined by

$$
\|v\|_{b}:=\left(\varepsilon|v|_{1}^{2}+\|v\|^{2}\right)^{1 / 2}
$$

Clearly, the balanced norm $\|\cdot\|_{b}$ is stronger than the energy norm $\|\cdot\|_{\varepsilon}$ in the case of $0<\varepsilon \ll 1$. Furthermore, the former is better suited to capture of layers. For example, for a typical layer function $e^{-x / \varepsilon},\left\|e^{-x / \varepsilon}\right\|_{\varepsilon}$ and $\left\|e^{-x / \varepsilon}\right\|_{b}$ are of order $\mathcal{O}\left(\varepsilon^{1 / 2}\right)$ and of order $\mathcal{O}(1)$, respectively. These orders imply that the balanced norm $\|\cdot\|_{b}$ is more appropriate to capture layers than the energy norm $\|\cdot\|_{\varepsilon}$ when $0<\varepsilon \ll 1$. The reader is also referred to [18, 4] for discussions on these two norms.

\section{Uniform convergence}

For convergence analysis in the balanced norm, we will present an interpolation operator, which components will be introduced at first.

Set $\Omega_{0}^{*}:=\left(x_{N / 4-1}, x_{3 N / 4+1}\right)^{2}$ and $\Omega_{0}^{* *}:=\left(x_{N / 4-2}, x_{3 N / 4+2}\right)^{2}$. Introduce a weighted $L^{2}$-projection $\pi$ as follows: for $s \in L^{2}\left(\Omega_{0}^{*}\right)$, find $\pi s \in W^{N}$ such that

$$
\left(b(s-\pi s), v^{N}\right)_{\Omega_{0}^{*}}=0 \quad \forall v^{N} \in W^{N},
$$

where $W^{N}=:\left\{\left.v\right|_{\Omega_{0}^{*}}: v \in V^{N}\right\}$. Of course, one has the $L^{2}$-stability

$$
\|\pi v\|_{\Omega_{0}^{*}} \leqslant C\|v\|_{\Omega_{0}^{*}}
$$

Denote by $\mathcal{I}$ the Langrange interpolation operator from $C^{0}(\bar{\Omega})$ to $V^{N}$. Furthermore, define $\chi \in V_{0}^{N}$ by

$$
\chi\left(s_{l}, t_{m}\right)= \begin{cases}1 & \left(s_{l}, t_{m}\right) \in \partial \Omega_{0}^{*}, \\ 0 & \text { otherwise }\end{cases}
$$

where $\left\{s_{l}, t_{m}\right\}$ are the interpolation points of the Lagrange interpolation. 
Recall $w=\sum_{i=1}^{4} w_{i}+\sum_{i=1}^{4} z_{i}$. Then the interpolation used in convergence analysis is defined by

$$
P_{c} u=P_{1} v_{0}+P_{2} w
$$

where

$$
P_{1} v_{0}:= \begin{cases}\pi v_{0} & \text { in } \Omega_{0}^{*}, \\ \mathcal{I}\left[(1-\chi) v_{0}+\chi \pi v_{0}\right] & \text { in } \Omega \backslash \Omega_{0}^{*}\end{cases}
$$

and

$$
P_{2} w:= \begin{cases}0 & \text { in } \Omega_{0}^{*}, \\ \mathcal{I}[(1-\chi) w] & \text { in } \Omega \backslash \Omega_{0}^{*} .\end{cases}
$$

Clearly, $P_{c} u \in V_{0}^{N}$.

The following lemma provides some pointwise bounds for errors between the Lagrange interpolant and the $L^{2}$ projection.

Lemma 2. For $v_{0}$ introduced in Assumption 1, one has

$$
\begin{aligned}
& \left\|\mathcal{I} v_{0}-\pi v_{0}\right\|_{\infty, \partial \Omega_{0}^{*}}+\left\|\mathcal{I} v_{0}-\pi v_{0}\right\|_{\infty, \Omega_{0}^{*}}+\left\|v_{0}-\pi v_{0}\right\|_{\infty, \Omega_{0}^{*}} \leqslant C N^{-(k+1)} \\
& \left\|\nabla\left(\mathcal{I} v_{0}-\pi v_{0}\right)\right\|_{\Omega_{0}} \leqslant C N^{-k}, \quad\left\|\nabla\left(\mathcal{I} v_{0}-\pi v_{0}\right)\right\|_{\Omega_{0}^{*} \backslash \Omega_{0}} \leqslant C \varepsilon^{-1 / 2} N^{-(k+1)}
\end{aligned}
$$

Proof. Standard interpolation theory and Lemma 1 imply $\left\|v_{0}-\mathcal{I} v_{0}\right\|_{\infty, \Omega_{0}^{*}}+\left\|v_{0}-\mathcal{I} v_{0}\right\|_{\Omega_{0}^{*}} \leqslant$ $C N^{-(k+1)}$. From the $L^{\infty}$-stability of the $L^{2}$-projection $\pi[5$, Theorem 1], one has

$$
\begin{aligned}
& \left\|\mathcal{I} v_{0}-\pi v_{0}\right\|_{\infty, \Omega_{0}^{*}}=\left\|\pi\left(\mathcal{I} v_{0}-v_{0}\right)\right\|_{\infty, \Omega_{0}^{*}} \leqslant C\left\|\mathcal{I} v_{0}-v_{0}\right\|_{\infty, \Omega_{0}^{*}} \leqslant C N^{-(k+1)}, \\
& \left\|\mathcal{I} v_{0}-\pi v_{0}\right\|_{\infty, \partial \Omega_{0}^{*}} \leqslant\left\|\mathcal{I} v_{0}-\pi v_{0}\right\|_{\infty, \Omega_{0}^{*}} \leqslant C N^{-(k+1)}, \\
& \left\|v_{0}-\pi v_{0}\right\|_{\infty, \Omega_{0}^{*}} \leqslant\left\|v_{0}-\mathcal{I} v_{0}\right\|_{\infty, \Omega_{0}^{*}}+\left\|\mathcal{I} v_{0}-\pi v_{0}\right\|_{\infty, \Omega_{0}^{*}} \leqslant C N^{-(k+1)} .
\end{aligned}
$$


Hölder inequalities, inverse inequalities, the $L^{\infty}$-stability of the $L^{2}$-projection [5, Theorem 1] and Lemma 1 yield

$$
\begin{aligned}
\left\|\nabla\left(\mathcal{I} v_{0}-\pi v_{0}\right)\right\|_{\Omega_{0}^{*} \backslash \Omega_{0}} & \leqslant \operatorname{meas}\left(\Omega_{0}^{*} \backslash \Omega_{0}\right)^{1 / 2}\left\|\nabla\left(\mathcal{I} v_{0}-\pi v_{0}\right)\right\|_{\infty, \Omega_{0}^{*} \backslash \Omega_{0}} \\
& \leqslant C h_{N / 4-1}^{-1 / 2}\left\|\mathcal{I} v_{0}-\pi v_{0}\right\|_{\infty, \Omega_{0}^{*} \backslash \Omega_{0}} \\
& =C h_{N / 4-1}^{-1 / 2}\left\|\pi\left(\mathcal{I} v_{0}-v_{0}\right)\right\|_{\infty, \Omega_{0}^{*}} \\
& \leqslant C h_{N / 4-1}^{-1 / 2}\left\|\mathcal{I} v_{0}-v_{0}\right\|_{\infty, \Omega_{0}^{*}} \\
& \leqslant C \varepsilon^{-1 / 2} N^{-(k+1)}
\end{aligned}
$$

Inverse inequalities and the $L^{2}$-stability of the $L^{2}$-projection (3.1) yield

$$
\begin{aligned}
& \left\|\nabla\left(\mathcal{I} v_{0}-\pi v_{0}\right)\right\|_{\Omega_{0}} \leqslant C N\left\|\mathcal{I} v_{0}-\pi v_{0}\right\|_{\Omega_{0}} \leqslant C N\left\|\mathcal{I} v_{0}-\pi v_{0}\right\|_{\Omega_{0}^{*}} \\
\leqslant & C N\left\|\mathcal{I} v_{0}-v_{0}\right\|_{\Omega_{0}^{*}} \leqslant C N^{-k} .
\end{aligned}
$$

Remark 1. Define

$$
V_{h}=\left\{v \in C\left[x_{N / 4-1}, x_{3 N / 4+1}\right] ;\left.v\right|_{\left(s_{j}, s_{j+1}\right)} \in P_{k}, j=0, \ldots, N / 2+1\right\}
$$

with $s_{j}=x_{N / 4-1+j}$ for $j=0, \ldots, N / 2+2$. In the same way as the proof of [5, Theorem 1], we could easily prove the $L^{\infty}$-stability of the $L^{2}$-projection $\pi_{h}: L^{2}\left(x_{N / 4-1}, x_{3 N / 4+1}\right) \rightarrow V_{h}$, that is

$$
\left\|\pi_{h} v\right\|_{\infty,\left(x_{N / 4-1}, x_{3 N / 4+1}\right)} \leqslant C\|v\|_{\infty,\left(x_{N / 4-1}, x_{3 N / 4+1}\right)} \quad \forall v \in L^{\infty}\left(x_{N / 4-1}, x_{3 N / 4+1}\right) .
$$

Furthermore, from tensor product we could easily obtain

$$
\|\pi v\|_{\infty, \Omega_{0}^{*}} \leqslant C\|v\|_{\infty, \Omega_{0}^{*}} \quad \forall v \in L^{\infty}\left(\Omega_{0}^{*}\right)
$$

The error $u-u^{N}$ is split as follows:

$$
u-u^{N}=\left(u-P_{c} u\right)+\left(P_{c} u-u^{N}\right)=: \eta+\xi .
$$


From the coercivity (2.17) and the Galerkin orthogonality (2.18) we have

$$
C\|\xi\|_{\varepsilon}^{2} \leqslant a\left(P_{c} u-u, \xi\right) \leqslant \varepsilon^{1 / 2}\|\eta\|_{b}\|\xi\|_{\varepsilon}+|(b \eta, \xi)| .
$$

In the following analysis, we give the estimations on each term in the right-hand side of (3.5).

Lemma 3. Let $\sigma \geqslant k+1$. Under Assumptions 1 and 2 we have

$$
|(b \eta, \xi)| \leqslant C \varepsilon^{1 / 2} N^{-(k+1)} \ln ^{1 / 2} N\|\xi\| .
$$

Proof. Our arguments are based on the following splitting

$$
\begin{aligned}
(b \eta, \xi)= & \left(b\left(v_{0}-P_{1} v_{0}\right), \xi\right)+\left(b\left(w-P_{2} w\right), \xi\right) \\
= & \left(b\left(v_{0}-\pi v_{0}\right), \xi\right)_{\Omega_{0}^{*}}+\left(b\left(v_{0}-\mathcal{I} v_{0}\right), \xi\right)_{\Omega \backslash \Omega_{0}^{*}}+\left(b \mathcal{I}\left[\chi\left(v_{0}-\pi v_{0}\right)\right], \xi\right)_{\Omega_{0}^{* *} \backslash \Omega_{0}^{*}} \\
& +(b w, \xi)_{\Omega_{0}^{*}}+(b(w-\mathcal{I} w), \xi)_{\Omega \backslash \Omega_{0}^{*}}+(b \mathcal{I}(\chi w), \xi)_{\Omega_{0}^{* *} \backslash \Omega_{0}^{*}} \\
= & : \mathrm{I}+\mathrm{II}+\mathrm{III}+\mathrm{IV}+\mathrm{V}+\mathrm{VI} .
\end{aligned}
$$

From the definition of $\pi$, we obtain

$$
\mathrm{I}=0 .
$$

From [24, Lemma 4], one has

$$
\begin{aligned}
|\mathrm{II}|+|\mathrm{V}| & \leqslant C\left(\left\|v_{0}-\mathcal{I} v_{0}\right\|_{\infty, \Omega \backslash \Omega_{0}^{*}}+\|w-\mathcal{I} w\|_{\infty, \Omega \backslash \Omega_{0}^{*}}\right)\|\xi\|_{1, \Omega \backslash \Omega_{0}^{*}} \\
& \leqslant C N^{-(k+1)} \operatorname{meas}^{1 / 2}\left(\Omega \backslash \Omega_{0}^{*}\right)\|\xi\|_{\Omega \backslash \Omega_{0}^{*}} \\
& \leqslant C \varepsilon^{1 / 2} N^{-(k+1)} \ln ^{1 / 2} N\|\xi\| .
\end{aligned}
$$

Lemmas 1 and 2, (2.3) and (2.4) yield

$$
\begin{aligned}
|\mathrm{III}|+|\mathrm{VI}| & \leqslant C\left(\left\|\mathcal{I} v_{0}-\pi v_{0}\right\|_{\infty, \partial \Omega_{0}^{*}}+\|\mathcal{I} w\|_{\infty, \partial \Omega_{0}^{*}}\right)\|\xi\|_{1, \Omega_{0}^{* *} \backslash \Omega_{0}^{*}} \\
& \leqslant C\left(N^{-(k+1)}+N^{-\sigma}\right) h_{N / 4-2}^{1 / 2}\|\xi\|_{\Omega_{0}^{* *} \backslash \Omega_{0}^{*}} \\
& \leqslant C \varepsilon^{1 / 2} N^{-(k+1)}\|\xi\| .
\end{aligned}
$$


From (2.3) and (2.4), we get

$$
|\mathrm{IV}| \leqslant C\|w\|_{\Omega_{0}^{*}}\|\xi\|_{\Omega_{0}^{*}} \leqslant C \varepsilon^{1 / 2} N^{-\sigma}\|\xi\|
$$

Collecting (3.6) $-(3.9)$, we are done.

Lemma 4. Let $\sigma \geqslant k+1$. Under Assumptions 1 and 0 we have

$$
\|\eta\|_{b} \leqslant C N^{-k}
$$

Proof. From (3.2), (3.3) and (3.4) one has

$$
\begin{aligned}
\|\eta\|_{b} \leqslant & \left\|v_{0}-\pi v_{0}\right\|_{b, \Omega_{0}^{*}}+\left\|v_{0}-\mathcal{I} v_{0}\right\|_{b, \Omega \backslash \Omega_{0}^{*}}+\left\|\mathcal{I}\left(\chi\left(v_{0}-\pi v_{0}\right)\right)\right\|_{b, \Omega_{0}^{* *} \backslash \Omega_{0}^{*}} \\
& +\|w-\mathcal{I} w\|_{b, \Omega \backslash \Omega_{0}^{*}}+\|\mathcal{I}(\chi w)\|_{b, \Omega_{0}^{* *} \backslash \Omega_{0}^{*}} \\
= & : S_{1}+S_{2}+S_{3}+S_{4}+S_{5} .
\end{aligned}
$$

To analyze $S_{1}$, we need the following bounds

$$
\begin{aligned}
\left\|\nabla\left(v_{0}-\pi v_{0}\right)\right\|_{\Omega_{0}} & \leqslant\left\|\nabla\left(v_{0}-\mathcal{I} v_{0}\right)\right\|_{\Omega_{0}}+\left\|\nabla\left(\mathcal{I} v_{0}-\pi v_{0}\right)\right\|_{\Omega_{0}} \leqslant C N^{-k}, \\
\left\|\nabla\left(v_{0}-\pi v_{0}\right)\right\|_{\Omega_{0}^{*} \backslash \Omega_{0}} & \leqslant\left\|\nabla\left(v_{0}-\mathcal{I} v_{0}\right)\right\|_{\Omega_{0}^{*} \backslash \Omega_{0}}+\left\|\nabla\left(\mathcal{I} v_{0}-\pi v_{0}\right)\right\|_{\Omega_{0}^{*} \backslash \Omega_{0}} \\
& \leqslant C N^{-k}+C \varepsilon^{-1 / 2} N^{-(k+1)} \\
\left\|v_{0}-\pi v_{0}\right\|_{\Omega_{0}^{*}} & \leqslant\left\|v_{0}-\mathcal{I} v_{0}\right\|_{\Omega_{0}^{*}}+\left\|\mathcal{I} v_{0}-\pi v_{0}\right\|_{\Omega_{0}^{*}} \\
& \leqslant C N^{-(k+1)}+C\left\|\mathcal{I} v_{0}-\pi v_{0}\right\|_{\infty, \Omega_{0}^{*}} \leqslant C N^{-(k+1)}
\end{aligned}
$$

which could be derived from standard interpolation theories and Lemma 2. Then we obtain

$$
\left|S_{1}\right| \leqslant \varepsilon^{1 / 2}\left\|\nabla\left(v_{0}-\pi v_{0}\right)\right\|_{\Omega_{0}^{*}}+\left\|v_{0}-\pi v_{0}\right\|_{\Omega_{0}^{*}} \leqslant C \varepsilon^{1 / 2} N^{-k}+C N^{-(k+1)} .
$$

Similar to [24, Lemma 4], we have

$$
\left\|v_{0}-\mathcal{I} v_{0}\right\|_{\infty, \Omega \backslash \Omega_{0}^{*}}+\|w-\mathcal{I} w\|_{\infty, \Omega \backslash \Omega_{0}^{*}} \leqslant C N^{-(k+1)} .
$$


Imitating the proof of Lemma 5 in [24] and replacing [24, (3.15)] by $\left|w_{1}\right|_{1,[0,1] \times\left[y_{N / 4-1}, 1\right]} \leqslant$ $\varepsilon^{-1 / 2} N^{-(k+1)}$, we obtain

$$
\left|w_{1}-\mathcal{I} w_{1}\right|_{1, \Omega \backslash \Omega_{0}^{*}} \leqslant C \varepsilon^{-1 / 2} N^{-k}
$$

and similarly have

$$
|w-\mathcal{I} w|_{1, \Omega \backslash \Omega_{0}^{*}} \leqslant C \varepsilon^{-1 / 2} N^{-k}
$$

Thus we obtain

$$
S_{2}+S_{4} \leqslant C N^{-k}
$$

Inverse inequalities, Lemmas 1 and 2 yield

$$
\begin{aligned}
S_{3} & \leqslant \varepsilon^{1 / 2}\left\|\nabla\left(\mathcal{I}\left(\chi\left(v_{0}-\pi v_{0}\right)\right)\right)\right\|_{\Omega_{0}^{* *} \backslash \Omega_{0}^{*}}+\left\|\mathcal{I}\left(\chi\left(v_{0}-\pi v_{0}\right)\right)\right\|_{\Omega_{0}^{* *} \backslash \Omega_{0}^{*}} \\
& \leqslant C\left(\varepsilon^{1 / 2} h_{N / 4-2}^{-1}+1\right)\left\|\mathcal{I}\left(\chi\left(v_{0}-\pi v_{0}\right)\right)\right\|_{\Omega_{0}^{* *} \backslash \Omega_{0}^{*}} \\
& \leqslant C\left(\varepsilon^{1 / 2} h_{N / 4-2}^{-1}+1\right) h_{N / 4-2}^{1 / 2}\left\|\mathcal{I} v_{0}-\pi v_{0}\right\|_{\infty, \partial \Omega_{0}^{*}} \\
& \leqslant C N^{-(k+1)} .
\end{aligned}
$$

Similarly, one has

$$
S_{5} \leqslant C N^{-\sigma}
$$

Collecting (3.10)- 3.13 , we are done.

Similar to [8, Theorem 2.6], we obtain the following theorem from Lemmas 3 and 4 .

Theorem 1. Let $\sigma \geqslant k+1$. Let Assumptions 1 and 2 hold. Then for the exact solution $u$ to (1.1) and the numerical solution $u^{N}$ to (2.16) on Bakhvalov-type rectangular mesh (2.5) or (2.6), one has

$$
\left\|u-u^{N}\right\|_{b} \leqslant C N^{-k}
$$




\section{Supercloseness}

In order to derive the supercloseness result in the balanced norm, we need another novel interpolant, which will be described in the following.

Instead of Langrange interpolant operator used in the previous section, we introduce an vertices-edges-element interpolation operator $\mathcal{A}: C^{0}(\bar{\Omega}) \rightarrow V^{N}$ (see [12, 21]). This interpolant is used for superconvergence analysis of the diffusion term. First we define the interpolant operator on the reference element $\hat{\tau}=(-1,1)^{2}$, whose vertices and edges are denoted by $\hat{a}_{i}$ and $\hat{e}_{i}$ respectively for $i=1, \ldots, 4$. Let $\hat{v}(\cdot, \cdot) \in C(\overline{\hat{\tau}})$. The operator $\hat{\mathcal{A}}: C(\overline{\hat{\tau}}) \rightarrow \mathcal{Q}_{k}(\hat{\tau})$ is determined by $(k+1)^{2}$ continuous linear functionals $\hat{F}: C(\overline{\hat{\tau}}) \rightarrow \mathbb{R}$, which are defined by

$$
\begin{aligned}
\hat{v} & \rightarrow \hat{v}\left(\hat{a}_{i}\right) \quad \mathrm{i}=1, \ldots, 4 \\
\hat{v} & \rightarrow \int_{\hat{e}_{i}} \hat{v} q \mathrm{~d} s \quad \forall q \in \mathcal{P}_{k-2}\left(\hat{e}_{i}\right) \quad \mathrm{i}=1, \ldots, 4 \\
\hat{v} & \rightarrow \int_{\hat{\tau}} \hat{v} q \mathrm{~d} x \mathrm{~d} y \quad \forall q \in \mathcal{Q}_{k-2}(\hat{\tau}) .
\end{aligned}
$$

From [21, Lemma 3], the operator $\hat{\mathcal{A}}$ is uniquely determined. Then using the affine transformation to map from $\hat{\tau}$ to an arbitrary $\tau \in \mathcal{T}^{N}$, one obtains the corresponding interpolation operator $\mathcal{A}_{\tau}: C(\bar{\tau}) \rightarrow \mathcal{Q}_{k}(\tau)$. At last a continuous global interpolation operator $\mathcal{A}: C(\bar{\Omega}) \rightarrow V^{N}$ is defined by setting

$$
\left.(\mathcal{A} v)\right|_{\tau}:=\mathcal{A}_{\tau}\left(\left.v\right|_{\tau}\right) \quad \forall \tau \in \mathcal{T}^{N}
$$

Besides, we denote by $F$ degree of freedom (DoF) of $V^{N}$, which originates from the linear functional $\hat{F}$.

Recall $i^{*}=j^{*}=N / 4-2$ and $\Omega_{w_{1}}:=[0,1] \times\left[y_{0}, y_{j^{*+1}}\right]$. The operator $S_{1}: C^{0}(\bar{\Omega}) \rightarrow$ $V^{N}$ is defined by

$$
S_{1} w_{1}:= \begin{cases}0 & \text { in } \Omega \backslash \Omega_{w_{1}}, \\ \mathcal{A} w_{1}-\mathcal{B}_{1} w_{1} & \text { in } \Omega_{w_{1}},\end{cases}
$$


where $\mathcal{B}_{1} w_{1}$ satisfies

$$
F\left(\mathcal{B}_{1} w_{1}\right)= \begin{cases}F\left(w_{1}\right) & \text { if } F \text { is the DoF of } V^{N} \text { attached to }[0,1] \times\left\{y=y_{j^{*}+1}\right\} \\ 0 & \text { otherwise. }\end{cases}
$$

If $F$ is the DoF of $V^{N}$ attached to $[0,1] \times\left\{y=y_{j^{*}+1}\right\}$, then it must be one of the following forms

$$
\begin{aligned}
& v \rightarrow v\left(x_{i}, y_{j^{*}+1}\right) \quad \text { for } i=0, \ldots, N \\
& v \rightarrow \frac{j+1}{h_{i}^{j+1}} \int_{x_{i}}^{x_{i+1}} v\left(s, y_{j^{*}+1}\right)\left(s-x_{i}\right)^{j} \mathrm{~d} s \text { for } i=0, \ldots, N-1 \text { and } j=0, \ldots, k-2 .
\end{aligned}
$$

In fact, $\mathcal{B}_{1} w_{1}$ is introduced for the continuity of $S_{1} w_{1}$ at $[0,1] \times\left\{y=y_{j^{*}+1}\right\}$. Set $\Omega_{z_{1}}:=$ $\left[x_{0}, x_{i^{*}+1}\right] \times\left[y_{0}, y_{j^{*}+1}\right]$. The operator $T_{1}: C^{0}(\bar{\Omega}) \rightarrow V^{N}$ is defined by

$$
T_{1} z_{1}:= \begin{cases}0 & \text { in } \Omega \backslash \Omega_{z_{1}}, \\ \mathcal{A} z_{1}-\mathcal{C}_{1} z_{1} & \text { in } \Omega_{z_{1}},\end{cases}
$$

where the operator $\mathcal{C}_{1}$ is defined in a similar way to $\mathcal{B}_{1}$ except that the degrees of freedom for $\mathcal{C}_{1}$ are attached to $\left[0, x_{i^{*}+1}\right] \times\left\{y=y_{j^{*}+1}\right\} \cup\left\{x=x_{i^{*}+1}\right\} \times\left[0, y_{j^{*+1}}\right]$. The operators $S_{i}$ and $T_{i}$ for $i=2,3,4$ could be defined similarly. Also we give a boundary correction $\mathcal{C}\left(S_{1} w_{1}\right) \in V^{N}$ for $S_{1} w_{1}$, which is defined by

$$
F\left(\mathcal{C}\left(S_{1} w_{1}\right)\right)= \begin{cases}F\left(w_{1}\right) & \text { if } F \text { is the DoF of } V^{N} \text { attached to } \Gamma_{w_{1}}, \\ 0 & \text { otherwise }\end{cases}
$$

where $\Gamma_{w_{1}}:=\overline{\partial \Omega \backslash \partial \Omega_{w_{1}}}$. With the help of this correction, we have

$$
\left.\left(S_{1} w_{1}+\mathcal{C}\left(S_{1} w_{1}\right)\right)\right|_{\partial \Omega}=\left.\mathcal{A} w_{1}\right|_{\partial \Omega}
$$

By the same token, we could define the corrections $\mathcal{C}\left(S_{i} w_{i}\right)$ and $\mathcal{C}\left(T_{i} z_{i}\right)$ for $S_{i} w_{i}$ and $T_{i} z_{i}$ for $i=1,2,3,4$, respectively.

Introduce the discrete function $\mathcal{D} v_{0} \in V_{0}^{N}$, which is defined by

$$
F\left(\mathcal{D} v_{0}\right)= \begin{cases}F\left(\pi v_{0}-v_{0}\right) & \text { if } F \text { is the DoF of } V^{N} \text { attached to } \partial \Omega_{0}^{*} \\ 0 & \text { otherwise }\end{cases}
$$


and

$$
\mathcal{E} v_{0}:= \begin{cases}\pi v_{0} & \text { in } \Omega_{0}^{*}, \\ \mathcal{A} v_{0}+\mathcal{D} v_{0} & \text { in } \Omega \backslash \Omega_{0}^{*} .\end{cases}
$$

Note that $\left.\left(\mathcal{D} v_{0}\right)\right|_{\partial \Omega_{0}^{*}}=\left.\left(\pi v_{0}-\mathcal{A} v_{0}\right)\right|_{\partial \Omega_{0}^{*}}$. The definition of $\mathcal{D} v_{0}$ ensures the continuity of $\mathcal{E} v_{0}$ on $\bar{\Omega}$.

Now we are in a position to propose the interpolation used for our supercloseness analysis, which is defined by

$$
P_{s} u=\mathcal{E} v_{0}+\sum_{i=1}^{4} S_{i} w_{i}+\sum_{i=1}^{4} T_{i} z_{i}+\mathcal{C}(w),
$$

where $\mathcal{C}(w)=\sum_{i=1}^{4} \mathcal{C}\left(S_{i} w_{i}\right)+\sum_{i=1}^{4} \mathcal{C}\left(T_{i} z_{i}\right)$. Clearly $P_{s} u \in V_{0}^{N}$

The following lemma could be found in [21, Lemma 4], which is important for analysis of the diffusion part.

Lemma 5. Let $\tau \in \mathcal{T}^{N}$. Let $v \in H^{k+2}(\tau)$ and $\mathcal{A}_{\tau} v \in \mathcal{Q}_{k}(\tau)$ be its vertices-edges-element interpolant. Then for each $v^{N} \in \mathcal{Q}_{k}(\tau)$ we have

$$
\left|\int_{\tau}\left(\mathcal{A}_{\tau} v-v\right)_{x} v_{x}^{N} \mathrm{~d} x \mathrm{~d} y\right| \leqslant C h_{y, \tau}^{k+1}\left\|\frac{\partial^{k+2} v}{\partial x \partial y^{k+1}}\right\|_{\tau}\left\|v_{x}^{N}\right\|_{\tau}
$$

and

$$
\left|\int_{\tau}\left(\mathcal{A}_{\tau} v-v\right)_{y} v_{y}^{N} \mathrm{~d} x \mathrm{~d} y\right| \leqslant C h_{x, \tau}^{k+1}\left\|\frac{\partial^{k+2} v}{\partial x^{k+1} \partial y}\right\|_{\tau}\left\|v_{y}^{N}\right\|_{\tau} .
$$

For the vertices-edges-element interpolation, we have the following interpolation errors.

Lemma 6. Let $\tau \in \mathcal{T}^{N}$. Let $v \in H^{k+1}(\Omega)$. Then there exists a constant $C$ such that the vertices-edges-element interpolation $\mathcal{A} v$ satisfies

$$
\|v-\mathcal{A} v\|_{\tau} \leqslant C \sum_{i+j=k+1} h_{x, \tau}^{i} h_{y, \tau}^{j}\left\|\frac{\partial^{k+1} v}{\partial x^{i} \partial y^{j}}\right\|_{\tau}
$$

Proof. See [21, Lemma 7]. 
Set

$$
e(D)=\left\{\tau \in \mathcal{T}^{N}: \bar{\tau} \cap(\overline{\Omega \backslash D}) \neq \varnothing, \tau \in D\right\}
$$

The following $L^{\infty}$-stabilities will be used later.

Lemma 7. Let $v \in C^{0}(\bar{\Omega})$. There exists a constant $C$ independent of $v$ such that

$$
\begin{aligned}
& \left\|\mathcal{B}_{1} v\right\|_{\infty, e\left(\Omega_{w_{1}}\right)} \leqslant C\|v\|_{\infty, e\left(\Omega_{w_{1}}\right)}, \\
& \left\|\left(\mathcal{B}_{1} v\right)_{x}\right\|_{\infty, e\left(\Omega_{w_{1}}\right)} \leqslant C\left\|v_{x}\right\|_{\infty, e\left(\Omega_{w_{1}}\right)}, \\
& \left\|\mathcal{C}_{1} z_{1}\right\|_{\infty, e\left(\Omega_{z_{1}}\right)} \leqslant C\left\|z_{1}\right\|_{\infty, e\left(\Omega_{z_{1}}\right)} .
\end{aligned}
$$

Proof. For $\tau_{i, j} *$ with $i=0, \ldots, N-1$, one has

$$
\begin{aligned}
\left.\mathcal{B}_{1} v\right|_{\tau_{i, j} *}= & v\left(x_{i}, y_{j^{*}+1}\right) \varphi_{i}(x, y)+v\left(x_{i+1}, y_{j^{*}+1}\right) \varphi_{i+1}(x, y) \\
& +\sum_{m=0}^{k-2} \frac{m+1}{h_{i, x}^{m+1}} \int_{x_{i}}^{x_{i+1}} v\left(s, y_{j^{*}+1}\right)\left(s-x_{i}\right)^{m} \mathrm{~d} s \varphi_{i, m}(x, y) .
\end{aligned}
$$

The inequality $\left\|\mathcal{B}_{1} v\right\|_{\infty, \tau_{i, j *}} \leqslant C\|v\|_{\infty, \tau_{i, j} *}$ follows. In a similar way, we could prove $\left.\left\|\mathcal{C}_{1} z_{1}\right\|_{\infty, e\left(\Omega_{z_{1}}\right)}\right) \leqslant C\left\|_{1}\right\|_{\infty, e\left(\Omega_{z_{1}}\right)}$. Note

$$
v\left(s, y_{j^{*}+1}\right)=v\left(x, y_{j^{*}+1}\right)+\int_{x}^{s} v_{t}\left(t, y_{j^{*}+1}\right) \mathrm{d} t \quad \text { for } s, x \in\left[x_{i}, x_{i+1}\right],
$$

and

$$
\left(\varphi_{i}(x, y)+\sum_{m=0}^{k-2} \varphi_{i, m}(x, y)+\varphi_{i+1}(x, y)\right)_{x} \equiv 0 \quad \text { for }(x, y) \in \tau_{i, j^{*}} .
$$

Then we have

$$
\begin{aligned}
\left.\left(\mathcal{B}_{1} v\right)_{x}\right|_{\tau_{i, j} *}= & \int_{x}^{x_{i}} v_{t}\left(t, y_{j^{*}+1}\right) \mathrm{d} t\left(\varphi_{i}(x, y)\right)_{x}+\int_{x}^{x_{i+1}} v_{t}\left(t, y_{j^{*}+1}\right) \mathrm{d} t\left(\varphi_{i+1}(x, y)\right)_{x} \\
& +\sum_{m=0}^{k-2} \frac{m+1}{h_{i, x}^{m+1}} \int_{x_{i}}^{x_{i+1}}\left(\int_{x}^{s} v_{t}\left(t, y_{j^{*}+1}\right) \mathrm{d} t\right)\left(s-x_{i}\right)^{m} \mathrm{~d} s\left(\varphi_{i, m}(x, y)\right)_{x}
\end{aligned}
$$

and

$$
\left\|\left(\mathcal{B}_{1} v\right)_{x}\right\|_{\infty, \tau_{i, j} *} \leqslant C M\left\|v_{x}\right\|_{\infty, \tau_{i, j *}} \leqslant C\left\|v_{x}\right\|_{\infty, \tau_{i, j} *}
$$


where from scaling arguments one has

$$
M=h_{i} \max _{\substack{l=i, i+1 \\ m=0, \ldots, k-2}}\left\{\left\|\left(\varphi_{l}(x, y)\right)_{x}\right\|_{\infty, \tau_{i, j^{*}}},\left\|\left(\varphi_{i, m}(x, y)\right)_{x}\right\|_{\infty, \tau_{i, j^{*}}}\right\} \leqslant C .
$$

Consider $e\left(\Omega_{w_{1}}\right)=\cup_{i=0}^{N-1} \tau_{i, j^{*}}$ and we are done.

The error is split as follows:

$$
u-u^{N}=\left(u-P_{s} u\right)+\left(P_{s} u-u^{N}\right)=: \tilde{\eta}+\tilde{\xi} .
$$

From the coercivity (2.17), the Galerkin orthogonality (2.18) and (4.4), one has

$$
\begin{aligned}
C\|\tilde{\xi}\|_{\varepsilon}^{2} & \leqslant a\left(P_{s} u-u, \tilde{\xi}\right)=\varepsilon^{2}(\nabla \tilde{\eta}, \nabla \tilde{\xi})+(b \tilde{\eta}, \tilde{\xi}) \\
& =: \varepsilon^{2} \mathscr{S}_{1}+\mathscr{S}_{2},
\end{aligned}
$$

where

$$
\begin{aligned}
& \mathscr{S}_{1}=\left(\nabla\left(v_{0}-\mathcal{E} v_{0}\right), \nabla \tilde{\xi}\right)+\sum_{i=1}^{4}\left(\nabla\left(w_{i}-S_{i} w_{i}\right), \nabla \tilde{\xi}\right)+\sum_{i=1}^{4}\left(\nabla\left(z_{i}-T_{i} z_{i}\right), \nabla \tilde{\xi}\right), \\
& \mathscr{S}_{2}=\left(b\left(v_{0}-\mathcal{E} v_{0}\right), \tilde{\xi}\right)+\sum_{i=1}^{4}\left(b\left(\left(w_{i}-S_{i} w_{i}\right)+\left(z_{i}-T_{i} z_{i}\right)\right), \tilde{\xi}\right)+a(\mathcal{C}(w), \tilde{\xi}) .
\end{aligned}
$$

The terms in the right-hand side of (4.5) will be analyzed in the following two lemmas.

Lemma 8. Let Assumptions 1 and 0 hold. Let $\sigma \geqslant k+3 / 2$. Then one has

$$
\left|\mathscr{S}_{1}\right| \leqslant C \varepsilon^{-3 / 2}\left(\varepsilon^{1 / 2} N^{-k}+N^{-(k+1)}\right)\|\tilde{\xi}\|_{\varepsilon}
$$

Proof. From Assumption 1, we just analyze $\left(\nabla\left(v_{0}-\mathcal{E} v_{0}\right), \nabla \tilde{\xi}\right),\left(\nabla\left(w_{1}-S_{1} w_{1}\right), \nabla \tilde{\xi}\right)$ and $\left(\left(z_{1}-T_{1} z_{1}\right)_{x}, \tilde{\xi}_{x}\right)$. The remaining terms can be treated in a similar manner. We split these terms as follows:

$$
\left(\nabla\left(v_{0}-\mathcal{E} v_{0}\right), \nabla \tilde{\xi}\right)+\left(\nabla\left(w_{1}-S_{1} w_{1}\right), \nabla \tilde{\xi}\right)+\left(\left(z_{1}-T_{1} z_{1}\right)_{x}, \tilde{\xi}_{x}\right)=\sum_{i=1}^{4} \mathcal{S}_{i}
$$


where

$$
\begin{aligned}
\mathcal{S}_{1} & =\left(\nabla\left(v_{0}-\mathcal{A} v_{0}\right), \nabla \tilde{\xi}\right)_{\Omega \backslash \Omega_{0}^{*}}+\left(\nabla\left(w_{1}-\mathcal{A} w_{1}\right), \nabla \tilde{\xi}\right)_{\Omega_{w_{1}}}+\left(\left(z_{1}-\mathcal{A} z_{1}\right)_{x}, \tilde{\xi}_{x}\right)_{\Omega_{z_{1}}}, \\
\mathcal{S}_{2} & =\left(\left(\mathcal{B}_{1} w_{1}\right)_{x}, \tilde{\xi}_{x}\right)_{e\left(\Omega_{w_{1}}\right)} \\
\mathcal{S}_{3} & =\left(\left(\mathcal{B}_{1} w_{1}\right)_{y}, \tilde{\xi}_{y}\right)_{e\left(\Omega_{w_{1}}\right)}+\left(\left(\mathcal{C}_{1} z_{1}\right)_{x}, \tilde{\xi}_{x}\right)_{e\left(\Omega_{z_{1}}\right)}, \\
\mathcal{S}_{4} & =\left(\nabla w_{1}, \nabla \tilde{\xi}\right)_{\Omega \backslash \Omega_{w_{1}}}+\left(\left(z_{1}\right)_{x}, \tilde{\xi}_{x}\right)_{\Omega \backslash \Omega_{z_{1}}}, \\
\mathcal{S}_{5} & =\left(\nabla\left(v_{0}-\pi v_{0}\right), \nabla \tilde{\xi}\right)_{\Omega_{0}^{*} \backslash \Omega_{0}}+\left(\nabla\left(v_{0}-\pi v_{0}\right), \nabla \tilde{\xi}\right)_{\Omega_{0}}, \\
\mathcal{S}_{6} & =\left(\nabla\left(\mathcal{D} v_{0}\right), \nabla \tilde{\xi}\right)_{\Omega \backslash \Omega_{0}^{*}} .
\end{aligned}
$$

Applying Lemmas 1 and 5 to $\mathcal{S}_{1}$, then we have

$$
\begin{aligned}
\left|\left(\left(w_{1}-\mathcal{A} w_{1}\right)_{x}, \tilde{\xi}_{x}\right)_{\Omega_{w_{1}}}\right| & \leqslant C \sum_{i=0}^{N-1} \sum_{j=0}^{j^{*}} h_{j, y}^{k+1}\left\|\frac{\partial^{k+2} w_{1}}{\partial x \partial^{k+1} y}\right\|_{\tau_{i j}}\left\|\tilde{\xi}_{x}\right\|_{\tau_{i j}} \\
& \leqslant C \sum_{i=0}^{N-1} \sum_{j=0}^{j^{*}} h_{j, y}^{k+1} \varepsilon^{-(k+1)} e^{-\beta y_{j} / \varepsilon} h_{i, x}^{1 / 2} h_{j, y}^{1 / 2}\left\|\tilde{\xi}_{x}\right\|_{\tau_{i j}} \\
& \leqslant C \varepsilon^{1 / 2} \sum_{i=0}^{N-1} \sum_{j=0}^{j^{*}} N^{-(k+2)}\left\|\tilde{\xi}_{x}\right\|_{\tau_{i j}} \\
& \leqslant C \varepsilon^{1 / 2} N^{-(k+1)}\left\|\tilde{\xi}_{x}\right\|
\end{aligned}
$$

and similarly obtain

$\left|\left(\nabla\left(v_{0}-\mathcal{A} v_{0}\right), \nabla \tilde{\xi}\right)_{\Omega \backslash \Omega_{0}^{*}}\right|+\left|\left(\left(w_{1}-\mathcal{A} w_{1}\right)_{y}, \tilde{\xi}_{y}\right)_{\Omega_{w_{1}}}\right|+\left|\left(\left(z_{1}-\mathcal{A} z_{1}\right)_{x}, \tilde{\xi}_{x}\right)_{\Omega_{z_{1}}}\right| \leqslant C \varepsilon^{-1 / 2} N^{-(k+1)}\|\nabla \tilde{\xi}\|$.

From Lemma 7, one has

$$
\begin{aligned}
\left|\mathcal{S}_{2}\right| & \leqslant\left\|\left(\mathcal{B}_{1} w_{1}\right)_{x}\right\|_{e\left(\Omega_{w_{1}}\right)}\|\nabla \tilde{\xi}\| \\
& \leqslant\left|e\left(\Omega_{w_{1}}\right)\right|^{1 / 2}\left\|\left(\mathcal{B}_{1} w_{1}\right)_{x}\right\|_{\infty, e\left(\Omega_{w_{1}}\right)}\|\nabla \tilde{\xi}\| \\
& \leqslant C \varepsilon^{1 / 2}\left\|\left(w_{1}\right)_{x}\right\|_{\infty, e\left(\Omega_{w_{1}}\right)}\|\nabla \tilde{\xi}\| \\
& \leqslant C \varepsilon^{1 / 2} N^{-\sigma}\|\nabla \tilde{\xi}\| .
\end{aligned}
$$


Inverse inequalities yield

$$
\begin{aligned}
\left|\mathcal{S}_{3}\right| & \leqslant\left(\left\|\left(\mathcal{B}_{1} w_{1}\right)_{y}\right\|_{e\left(\Omega_{w_{1}}\right)}+\left\|\left(\mathcal{C}_{1} z_{1}\right)_{x}\right\|_{e\left(\Omega_{z_{1}}\right)}\right)\|\nabla \tilde{\xi}\| \\
& \leqslant C\left(h_{j^{*}, y}^{-1}\left\|\mathcal{B}_{1} w_{1}\right\|_{e\left(\Omega_{w_{1}}\right)}+h_{i^{*}, x}^{-1}\left\|\mathcal{C}_{1} z_{1}\right\|_{e\left(\Omega_{z_{1}}\right)}\right)\|\nabla \tilde{\xi}\| \\
& \leqslant C \varepsilon^{-1}\left(\left|e\left(\Omega_{w_{1}}\right)\right|^{1 / 2}\left\|\mathcal{B}_{1} w_{1}\right\|_{\infty, e\left(\Omega_{w_{1}}\right)}+\left|e\left(\Omega_{z_{1}}\right)\right|^{1 / 2}\left\|\mathcal{C}_{1} z_{1}\right\|_{\infty, e\left(\Omega_{z_{1}}\right)}\right)\|\nabla \tilde{\xi}\| \\
& \leqslant C \varepsilon^{-1 / 2}\left(\left\|w_{1}\right\|_{\infty, e\left(\Omega_{w_{1}}\right)}+\left\|z_{1}\right\|_{\infty, e\left(\Omega_{z_{1}}\right)}\right)\|\nabla \tilde{\xi}\| \\
& \leqslant C \varepsilon^{-1 / 2} N^{-\sigma}\|\nabla \tilde{\xi}\| .
\end{aligned}
$$

Assumption 1 and direct calculations yield

$$
\left|\mathcal{S}_{4}\right| \leqslant\left(\left\|\nabla w_{1}\right\|_{\Omega \backslash \Omega_{w_{1}}}+\left\|\nabla z_{1}\right\|_{\Omega \backslash \Omega_{z_{1}}}\right)\|\nabla \tilde{\xi}\| \leqslant C \varepsilon^{-1 / 2} N^{-\sigma}\|\nabla \tilde{\xi}\| .
$$

Hölder inequalities, inverse inequalities, Lemmas 1 and 2 yield

$$
\begin{aligned}
\left|\mathcal{S}_{5}\right| & \leqslant C h_{N / 4-1}^{-1}\left\|v_{0}-\pi v_{0}\right\|_{\infty, \Omega_{0}^{*} \backslash \Omega_{0}}\|\nabla \tilde{\xi}\|_{1, \Omega_{0}^{*} \backslash \Omega_{0}}+C N\left\|v_{0}-\pi v_{0}\right\|_{\infty, \Omega_{0}}\|\nabla \tilde{\xi}\|_{1, \Omega_{0}} \\
& \leqslant C h_{N / 4-1}^{-1} N^{-(k+1)} h_{N / 4-1}^{1 / 2}\|\nabla \tilde{\xi}\|_{\Omega_{0}^{*} \backslash \Omega_{0}}+C N N^{-(k+1)}\|\nabla \tilde{\xi}\|_{\Omega_{0}} \\
& \leqslant C \varepsilon^{-3 / 2}\left(N^{-(k+1)}+\varepsilon^{1 / 2} N^{-k}\right)\|\tilde{\xi}\|_{\varepsilon}
\end{aligned}
$$

From the definition of $\mathcal{D} v_{0}$, we have

$$
\begin{aligned}
\left|\mathcal{S}_{6}\right| & \leqslant C\left\|\nabla\left(\mathcal{D} v_{0}\right)\right\|_{\infty, \partial \Omega_{0}^{*}}\|\nabla \tilde{\xi}\|_{1, e\left(\Omega \backslash \Omega_{0}^{*}\right)} \\
& \leqslant C h_{N / 4-2}^{-1}\left\|\mathcal{A} v_{0}-\pi v_{0}\right\|_{\infty, \partial \Omega_{0}^{*}} \cdot h_{N / 4-2}^{1 / 2}\|\nabla \tilde{\xi}\|_{e\left(\Omega \backslash \Omega_{0}^{*}\right)} \\
& \leqslant C \varepsilon^{-3 / 2}\left\|\mathcal{A} v_{0}-\pi v_{0}\right\|_{\infty, \Omega_{0}^{*}}\|\tilde{\xi}\|_{\varepsilon} \\
& \leqslant C \varepsilon^{-3 / 2} N^{-(k+1)}\|\tilde{\xi}\|_{\varepsilon} .
\end{aligned}
$$

where Lemmas 2 and 6 yield

$$
\left\|\mathcal{A} v_{0}-\pi v_{0}\right\|_{\infty, \Omega_{0}^{*}} \leqslant\left\|\mathcal{A} v_{0}-v_{0}\right\|_{\infty, \Omega_{0}^{*}}+\left\|v_{0}-\pi v_{0}\right\|_{\infty, \Omega_{0}^{*}} \leqslant C N^{-(k+1)} .
$$

Substituting (4.7)-(4.13) into (4.6) and considering $\sigma \geqslant k+3 / 2$, we are done.

Lemma 9. Let Assumptions 1 and 0 hold. Let $\sigma \geqslant k+3 / 2$. Then one has

$$
\left|\mathscr{S}_{2}\right| \leqslant C \varepsilon^{1 / 2} N^{-(k+1)} \ln ^{1 / 2} N\|\tilde{\xi}\|_{\varepsilon}
$$


Proof. From (4.5), we have

$$
\mathscr{S}_{2}=\left(b\left(v_{0}-\mathcal{E} v_{0}\right), \tilde{\xi}\right)+\sum_{i=1}^{4}\left(b\left(w_{i}-S_{i} w_{i}\right), \tilde{\xi}\right)+\sum_{i=1}^{4}\left(b\left(z_{i}-T_{i} z_{i}\right), \tilde{\xi}\right)+a(\mathcal{C}(w), \tilde{\xi}) .
$$

First, from (4.3), Hölder inequalities and Lemma 1 we obtain

$$
\begin{aligned}
& \left|\left(b\left(v_{0}-\mathcal{E} v_{0}\right), \tilde{\xi}\right)\right| \leqslant\left|\left(b\left(v_{0}-\mathcal{A} v_{0}\right), \tilde{\xi}\right)_{\Omega \backslash \Omega_{0}^{*}}\right|+\left|\left(b\left(\mathcal{D} v_{0}\right), \tilde{\xi}\right)_{\Omega \backslash \Omega_{0}^{*}}\right| \\
& \leqslant C\left\|v_{0}-\mathcal{A} v_{0}\right\|_{\infty, \Omega \backslash \Omega_{0}^{*}}\|\tilde{\xi}\|_{1, \Omega \backslash \Omega_{0}^{*}}+C\left\|\mathcal{A} v_{0}-\pi v_{0}\right\|_{\infty, \partial \Omega_{0}^{*}}\|\tilde{\xi}\|_{1, e\left(\Omega \backslash \Omega_{0}^{*}\right)} \\
& \leqslant C \varepsilon^{1 / 2} N^{-(k+1)} \ln ^{1 / 2} N\|\tilde{\xi}\|_{\Omega \backslash \Omega_{0}^{*}}+C N^{-(k+1)} h_{N / 4-2}^{1 / 2}\|\tilde{\xi}\|_{e\left(\Omega \backslash \Omega_{0}^{*}\right)} .
\end{aligned}
$$

Second, Hölder inequalities and Lemma 1 yield

$$
\begin{aligned}
& a(\mathcal{C}(w), \tilde{\xi}) \leqslant \varepsilon^{2}|(\nabla \mathcal{C}(w), \nabla \tilde{\xi})|+|(b \mathcal{C}(w), \tilde{\xi})| \\
\leqslant & C \varepsilon^{2}\|\nabla \mathcal{C}(w)\|_{\infty, e(\Omega)}\|\nabla \tilde{\xi}\|_{1, e(\Omega)}+C\|\mathcal{C}(w)\|_{\infty, e(\Omega)}\|\tilde{\xi}\|_{1, e(\Omega)} \\
\leqslant & C \varepsilon^{2} h_{0}^{-1} N^{-\sigma} \cdot h_{0}^{1 / 2}\|\nabla \tilde{\xi}\|_{e(\Omega)}+C N^{-\sigma} \cdot h_{0}^{1 / 2}\|\tilde{\xi}\|_{e(\Omega)} \\
\leqslant & C \varepsilon^{1 / 2} N^{-(\sigma-1 / 2)}\|\tilde{\xi}\|_{\varepsilon}+C \varepsilon^{1 / 2} N^{-(\sigma+1 / 2)}\|\tilde{\xi}\|_{\varepsilon},
\end{aligned}
$$

where we have used $\|\mathcal{C}(w)\|_{\infty, e(\Omega)} \leqslant C N^{-\sigma}$ and $C_{6} \varepsilon N^{-1} \leqslant h_{0} \leqslant C_{7} \varepsilon N^{-1}$.

At last, we analyze $\left(b\left(w_{1}-S_{1} w_{1}\right), \tilde{\xi}\right)$ and the remaining terms can be discussed in a similar way. Lemmas 6 and 1 yield

$$
\begin{aligned}
& \left\|w_{1}-\mathcal{A} w_{1}\right\|_{\Omega_{w_{1}}}^{2}=\sum_{i=0}^{N-1} \sum_{j=0}^{j^{*}}\left\|w_{1}-\mathcal{A} w_{1}\right\|_{\tau_{i, j}}^{2} \\
\leqslant & C \sum_{i=0}^{N-1} \sum_{j=0}^{j^{*}}\left(\sum_{l+m=k+1} h_{i, x}^{l} h_{j, y}^{m}\left\|\frac{\partial^{k+1} w_{1}}{\partial x^{l} \partial y^{m}}\right\|_{\tau_{i, j}}\right)^{2} \\
\leqslant & C \sum_{i=0}^{N-1} \sum_{j=0}^{j^{*}}\left(\sum_{l+m=k+1} h_{i, x}^{l} h_{j, y}^{m} \varepsilon^{-m} e^{-\beta y_{j} / \varepsilon} h_{i, x}^{1 / 2} h_{j, y}^{1 / 2}\right)^{2} \\
\leqslant & C \sum_{i=0}^{N-1} \sum_{j=0}^{j^{*}}\left(\sum_{l+m=k+1} N^{-(l+1 / 2)} \varepsilon^{m+1 / 2} N^{-(m+1 / 2)} \varepsilon^{-m}\right)^{2} \\
\leqslant & C \sum_{i=0}^{N-1} \sum_{j=0}^{j^{*}}\left(\varepsilon^{1 / 2} N^{-(k+2)}\right)^{2} \leqslant C \varepsilon N^{-2(k+1)} .
\end{aligned}
$$


Lemma 7 yields

$$
\left\|\mathcal{B}_{1} w_{1}\right\|_{e\left(\Omega_{w_{1}}\right)} \leqslant C\left|e\left(\Omega_{w_{1}}\right)\right|^{1 / 2}\left\|w_{1}\right\|_{\infty, e\left(\Omega_{w_{1}}\right)} \leqslant C h_{j^{*}}^{1 / 2} N^{-\sigma}
$$

From the Cauchy-Schwarz inequality one obtains

$$
\begin{aligned}
& \left(b\left(w_{1}-S_{1} w_{1}\right), \tilde{\xi}\right)=\left(b w_{1}, \tilde{\xi}\right)_{\Omega \backslash \Omega_{w_{1}}}+\left(b\left(w_{1}-\mathcal{A} w_{1}\right), \tilde{\xi}\right)_{\Omega_{w_{1}}}+\left(b \mathcal{B}_{1} w_{1}, \tilde{\xi}\right)_{e\left(\Omega_{w_{1}}\right)} \\
\leqslant & C\left\|w_{1}\right\|_{\Omega \backslash \Omega_{w_{1}}}\|\tilde{\xi}\|_{\Omega \backslash \Omega_{w_{1}}}+C\left\|w_{1}-\mathcal{A} w_{1}\right\|_{\Omega_{w_{1}}}\|\tilde{\xi}\|_{\Omega_{w_{1}}}+\left\|\mathcal{B}_{1} w_{1}\right\|_{e\left(\Omega_{w_{1}}\right)}\|\tilde{\xi}\|_{e\left(\Omega_{w_{1}}\right)} \\
\leqslant & C\left(\varepsilon^{1 / 2} N^{-\sigma}+\varepsilon^{1 / 2} N^{-(k+1)}+C h_{j^{*}}^{1 / 2} N^{-\sigma}\right)\|\tilde{\xi}\|_{\varepsilon} \\
\leqslant & C \varepsilon^{1 / 2} N^{-(k+1)}\|\tilde{\xi}\|_{\varepsilon} .
\end{aligned}
$$

Substituting (4.15)-(4.17) into (4.14), we are done.

Now we are in a position to present our supercloseness result in the balanced norm.

Theorem 2. Let Assumptions 1 and 0 hold. Let $\sigma \geqslant k+3 / 2$. Let $P_{s} u$ defined in (4.4) be the interpolation to the solution $u$ of (1.1). Let $u^{N}$ be the solution of (2.14). Then one has

$$
\left\|P_{s} u-u^{N}\right\|_{b} \leqslant C\left(\varepsilon^{1 / 2} N^{-k}+N^{-(k+1)} \ln ^{1 / 2} N\right) .
$$

Proof. From (4.5), Lemmas 8 and 9, we obtain

$$
\|\tilde{\xi}\|_{\varepsilon} \leqslant C \varepsilon^{1 / 2}\left(\varepsilon^{1 / 2} N^{-k}+N^{-(k+1)} \ln ^{1 / 2} N\right)
$$

which implies the following estimations

$$
\begin{aligned}
& \varepsilon^{1 / 2}\left\|\nabla\left(P_{s} u-u^{N}\right)\right\| \leqslant C\left(\varepsilon^{1 / 2} N^{-k}+N^{-(k+1)} \ln ^{1 / 2} N\right), \\
& \left\|P_{s} u-u^{N}\right\| \leqslant C \varepsilon^{1 / 2}\left(\varepsilon^{1 / 2} N^{-k}+N^{-(k+1)} \ln ^{1 / 2} N\right) .
\end{aligned}
$$

Thus we are done.

Remark 2. From Theorems 1 and 2, we could conclude that Theorem 2 presents a supercloseness result. It is the first supercloseness result in the balanced norm in the literature. 


\section{Numerical experiment}

In this section we present numerical experiments on Bakhvaolv-type rectangular meshes that support our theoretical results. All calculations were carried out by using Intel Visual Fortran 11 and the discrete problems were solved using GMRES (see, e.g., [2]).

We present errors and convergence orders in the computed solutions for the boundary value problem

$$
\begin{aligned}
-\varepsilon^{2} \Delta u+2 u & =f(x, y) & & \text { in } \Omega=(0,1)^{2}, \\
u & =0 & & \text { on } \partial \Omega,
\end{aligned}
$$

where the right-hand side $f$ is chosen in such a way that

$$
u(x, y)=\left(1-\frac{e^{-x / \varepsilon}+e^{-(1-x) / \varepsilon}}{1+e^{-1 / \varepsilon}}\right)\left(1-\frac{e^{-y / \varepsilon}+e^{-(1-y) / \varepsilon}}{1+e^{-1 / \varepsilon}}\right)
$$

is the exact solution. For the computations we will assign values to the parameters in (2.5) and (2.6). We set $\sigma=k+1$ when the error $\left\|u-u^{N}\right\|_{b}$ is discussed and $\sigma=k+3 / 2$ when the error $\left\|P_{s} u-u^{N}\right\|_{b}$ is discussed. Also, we set $\beta=1$ and $C_{1}=4 \sigma /(3 \beta)$ in (2.6).

In our numerical tests, we will consider $\varepsilon=10^{-3}, 10^{-4}, \cdots, 10^{-6}, k=1,2,3$ and $N=12,24, \cdots, 768$. Our numerical experiments imply that meshes (2.5) and (2.6) have same performances. Thus we only present numerical results for mesh (2.5).

For a fixed $\varepsilon$ and $N$, we have evaluated the error $e_{c}^{N, \varepsilon}=\left\|u-u^{N}\right\|_{b}$ and $e_{s}^{N, \varepsilon}=\| P_{s} u-$ $u^{N} \|_{b}$, where $P_{s} u$ is the interpolation of the exact solution $u$ to (5.1), which is defined in (4.4) and $u^{N}$ represents its numerical approximation. In the following we present the errors

$$
e_{c}^{N}=\max _{\varepsilon=10^{-3}, \ldots, 10^{-6}} e_{c}^{N, \varepsilon}, \quad e_{s}^{N}=\max _{\varepsilon=10^{-3}, \ldots, 10^{-6}} e_{s}^{N, \varepsilon}
$$

and the corresponding orders of convergence

$$
p_{c}^{N}=\frac{\ln e_{c}^{N}-\ln e_{c}^{2 N}}{\ln 2}, \quad p_{s}^{N}=\frac{\ln e_{s}^{N}-\ln e_{s}^{2 N}}{\ln 2}
$$

Numerical results are presented in Table 1 and log-log chart 1. Table 1 lists errors in the balanced norm on Bakhvalov-type meshes (2.5) for $\varepsilon=10^{-3}, \ldots, 10^{-6}$ in the cases of 
$k=1$ and $k=2$. Errors in the balanced norm for the cases $k=3$ are plotted in Figure 1. The data in Table 1 and Figure 1 show optimal uniform convergence of $\left\|u-u^{N}\right\|_{b}$ with respect to the singular perturbation parameter $\varepsilon$, which implies that Theorem 1 is sharp. The data also show that the superclosenss result is of order $k+1$ for $k$ th rectangular finite elements, which support Theorem 2 in a sense and also imply Theorem 2 might not be sharp.

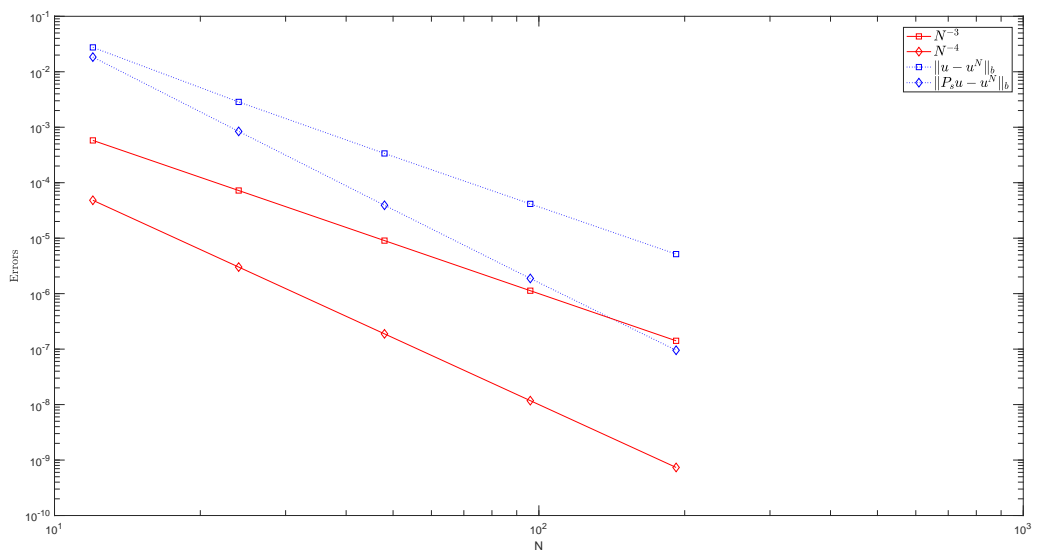

Fig.1: Errors in the balanced norm when $k=3$.

Table 1: Errors and rates in the balanced norm for $k=1$ and $k=2$

\begin{tabular}{c|cc|cc|cc|cc|}
\hline \multicolumn{8}{|c}{ Table 1: Errors and rates in the balanced norm for $k=1$ and $k=2$} \\
\hline$N$ & $k=1$ & $k=1$ & $k=1$ & $k=1$ & $k=2$ & $k=2$ & $k=2$ & $k=2$ \\
\hline 12 & $0.397 \mathrm{E} 0$ & 1.04 & $0.101 \mathrm{E} 0$ & 2.05 & $0.103 \mathrm{E} 0$ & 2.13 & $0.336 \mathrm{E}-1$ & 3.43 \\
24 & $0.193 \mathrm{E} 0$ & 1.00 & $0.245 \mathrm{E}-1$ & 2.11 & $0.236 \mathrm{E}-1$ & 2.03 & $0.312 \mathrm{E}-2$ & 3.40 \\
48 & $0.963 \mathrm{E}-1$ & 1.00 & $0.566 \mathrm{E}-2$ & 2.07 & $0.576 \mathrm{E}-2$ & 2.01 & $0.295 \mathrm{E}-3$ & 3.36 \\
96 & $0.481 \mathrm{E}-1$ & 1.00 & $0.135 \mathrm{E}-2$ & 2.04 & $0.143 \mathrm{E}-2$ & 2.00 & $0.287 \mathrm{E}-4$ & 3.29 \\
192 & $0.241 \mathrm{E}-1$ & 1.01 & $0.328 \mathrm{E}-3$ & 2.02 & $0.357 \mathrm{E}-3$ & 2.00 & $0.295 \mathrm{E}-5$ & 3.16 \\
384 & $0.120 \mathrm{E}-1$ & 1.00 & $0.808 \mathrm{E}-4$ & 2.01 & $0.892 \mathrm{E}-4$ & - & $0.328 \mathrm{E}-6$ \\
768 & $0.601 \mathrm{E}-2$ & - & $0.201 \mathrm{E}-4$ & - & - & - & - \\
\hline
\end{tabular}




\section{References}

\section{References}

[1] N. S. Bahvalov. On the optimization of the methods for solving boundary value problems in the presence of a boundary layer. Zh. Vychisl. Mat. Mat. Fiz., 9:841859, 1969.

[2] M. Benzi, G. H. Golub, and J. Liesen. Numerical solution of saddle point problems. Acta Numer., 14:1-137, 2005.

[3] C. Clavero, J. L. Gracia, and E. O'Riordan. A parameter robust numerical method for a two dimensional reaction-diffusion problem. Math. Comp., 74(252):1743-1758, 2005.

[4] P. Constantinou, S. Franz, L. Ludwig, and C. Xenophontos. Finite element approximation of reaction-diffusion problems using an exponentially graded mesh. Comput. Math. Appl., 76(10):2523-2534, 2018.

[5] M. Crouzeix and V. Thomée. The stability in $L_{p}$ and $W_{p}^{1}$ of the $L_{2}$-projection onto finite element function spaces. Math. Comp., 48(178):521-532, 1987.

[6] R. G. Durán, A. L. Lombardi, and M. I. Prieto. Supercloseness on graded meshes for $Q_{1}$ finite element approximation of a reaction-diffusion equation. J. Comput. Appl. Math., 242:232-247, 2013.

[7] S. Franz. Singularly perturbed problems with characteristic layers: Supercloseness and postprocessing. PhD thesis, Department of Mathematics, TU Dresden, 2008.

[8] S. Franz and H.-G. Roos. Error estimates in balanced norms of finite element methods for higher order reaction-diffusion problems, 2019.

[9] H. Han and R. B. Kellogg. Differentiability properties of solutions of the equation $-\epsilon^{2} \Delta u+r u=f(x, y)$ in a square. SIAM J. Math. Anal., 21(2):394-408, 1990. 
[10] N. Kopteva. On the convergence, uniform with respect to the small parameter, of a scheme with central difference on refined grids. Zh. Vychisl. Mat. Mat. Fiz., 39(10):1662-1678, 1999.

[11] N. Kopteva and S. B. Savescu. Pointwise error estimates for a singularly perturbed time-dependent semilinear reaction-diffusion problem. IMA J. Numer. Anal., 31(2):616-639, 2011.

[12] Q. Lin, N. Yan, and A. Zhou. A rectangle test for interpolated finite elements. In Proceedings Systems Science and Systems Engineering (Hong Kong, 1991), pages 217-229. Great Wall Culture Publishing, Whittier, CA, 1991.

[13] R. Lin and M. Stynes. A balanced finite element method for singularly perturbed reaction-diffusion problems. SIAM J. Numer. Anal., 50(5):2729-2743, 2012.

[14] T. Linß. Layer-adapted meshes for reaction-convection-diffusion problems, volume 1985 of Lecture Notes in Mathematics. Springer-Verlag, Berlin, 2010.

[15] F. Liu, N. Madden, M. Stynes, and A. Zhou. A two-scale sparse grid method for a singularly perturbed reaction-diffusion problem in two dimensions. IMA J. Numer. Anal., 29(4):986-1007, 2009.

[16] X. Liu, M. Stynes, and J. Zhang. Supercloseness of edge stabilization on Shishkin rectangular meshes for convection-diffusion problems with exponential layers. IMA J. Numer. Anal., 38(4):2105-2122, 2018.

[17] H.-G. Roos. Error estimates for linear finite elements on Bakhvalov-type meshes. Appl. Math., 51(1):63-72, 2006.

[18] H.-G. Roos and M. Schopf. Convergence and stability in balanced norms of finite element methods on Shishkin meshes for reaction-diffusion problems. ZAMM Z. Angew. Math. Mech., 95(6):551-565, 2015. 
[19] H.-G. Roos, M. Stynes, and L. Tobiska. Robust Numerical Methods for Singularly Perturbed Differential Equations, volume 24 of Springer Series in Computational Mathematics. Springer-Verlag, Berlin, second edition, 2008.

[20] M. Stynes. Steady-state convection-diffusion problems. Acta Numer., 14:445-508, 2005.

[21] M. Stynes and L. Tobiska. Using rectangular $Q_{p}$ elements in the SDFEM for a convection-diffusion problem with a boundary layer. Appl. Numer. Math., 58(12):1789-1802, 2008.

[22] J. Zhang and X. Liu. Analysis of SDFEM on Shishkin triangular meshes and hybrid meshes for problems with characteristic layers. J. Sci. Comput., 68(3):1299-1316, 2016.

[23] J. Zhang and X. Liu. Supercloseness of the SDFEM on Shishkin triangular meshes for problems with exponential layers. Adv. Comput. Math., 43(4):759-775, 2017.

[24] J. Zhang and X. Liu. Convergence of a finite element method on a Bakhvalov-type mesh for singularly perturbed reaction-diffusion equation. Appl. Math. Comput., $385: 125403,2020$.

[25] J. Zhang and X. Liu. Optimal order of uniform convergence for finite element method on Bakhvalov-type meshes. J. Sci. Comput., 85(1):2, 2020.

[26] J. Zhang and X. Liu. Supercloseness of linear finite element method on Bakhvalovtype meshes for singularly perturbed convection-diffusion equation in 1D. Appl. Math. Lett., 111:106624, 2021.

[27] J. Zhang and M. Stynes. Supercloseness of continuous interior penalty method for convection-diffusion problems with characteristic layers. Comput. Methods Appl. Mech. Engrg., 319:549-566, 2017. 